\title{
Beneficial effects of low-digestible carbohydrates in ulcerative colitis
}

A prebiotic low-digestible carbohydrate has been shown to have beneficial effects in a piglet model of ulcerative colitis.

Low-digestible carbohydrates seem to be important in maintaining normal bowel function and they induce the formation of short-chain fatty acids, such as butyrate, which is protective against IBD.

A randomized, placebo-controlled, parallel blind study was conducted to investigate the effects of a prebiotic lowdigestible carbohydrate on ulcerative colitis. 32 piglets were randomly assigned to receive prebiotic low-digestible carbohydrate or placebo for 44 days before colitis was induced.

Serum IgA levels, inflammatory protein profiles and the development of colitis were monitored. The microbiota profile was determined using real-time quantitative PCR.

"Prior and continuing low-digestible carbohydrate supplementation alleviated the symptoms of colitis (body weight loss, bloody stools)," reports Phillipe Pouillart, lead author of the study. In addition, proinflammatory factors were downregulated and there was a beneficial shift in the microbiota profile to include butyrogenic bacteria strains.

"At the dose tested, low-digestible carbohydrates are well-tolerated prebiotic agents able to not only stimulate butyrogenic bacteria strains ... but also to concomitantly prevent chronic inflammatory intestinal injuries," says Pouillart. "The current study thus confirms the role of low-digestible carbohydrates in the prevention and treatment of intestinal inflammation," the authors conclude.

Isobel Franks

Original article Pouillart, P. R. et al. Nutriose, a prebiotic low-digestible carbohydrate, stimulates gut mucosal immunity and prevent TNBS-induced colitis in piglets. Inflamm. Bowel Dis. 16, 783-794 (2010) 